\title{
The far-infrared/submillimeter properties of galaxies located behind the Bullet cluster ${ }^{\star, \star \star}$
}

\author{
M. Rex ${ }^{1}$, T. D. Rawle ${ }^{1}$, E. Egami ${ }^{1}$, P. G. Pérez-González ${ }^{2,1}$, M. Zemcov $^{3,4}$, I. Aretxaga ${ }^{5}$, S. M. Chung ${ }^{6}$, D. Fadda ${ }^{7}$, \\ A. H. Gonzalez ${ }^{6}$, D. H. Hughes ${ }^{5}$, C. Horellou ${ }^{8}$, D. Johansson ${ }^{8}$, J.-P. Kneib ${ }^{9}$, J. Richard ${ }^{10}$, B. Altieri ${ }^{11}$, A. K. Fiedler ${ }^{1}$, \\ M. J. Pereira ${ }^{1}$, G. H. Rieke ${ }^{1}$, I. Smail ${ }^{10}$, I. Valtchanov ${ }^{11}$, A. W. Blain ${ }^{3}$, J. J. Bock ${ }^{3,4}$, F. Boone ${ }^{12,13}$, C. R. Bridge $^{3}$, \\ B. Clement ${ }^{9}$, F. Combes ${ }^{13}$, C. D. Dowell ${ }^{3,4}$, M. Dessauges-Zavadsky ${ }^{14}$, O. Ilbert ${ }^{9}$, R. J. Ivison ${ }^{15,16}$, M. Jauzac ${ }^{9}$, \\ D. Lutz $^{17}$, A. Omont ${ }^{18}$, R. Pelló ${ }^{12}$, G. Rodighiero ${ }^{19}$, D. Schaerer ${ }^{14,12}$, G. P. Smith ${ }^{20}$, G. L. Walth ${ }^{1}$, P. van der Werf ${ }^{21}$, \\ M. W. Werner ${ }^{4}$, J. E. Austermann ${ }^{22}$, H. Ezawa ${ }^{23}$, R. Kawabe ${ }^{23}$, K. Kohno ${ }^{24,25}$, T. A. Perera ${ }^{26}$, K. S. Scott ${ }^{27}$, \\ G. W. Wilson ${ }^{28}$, and M. S. Yun ${ }^{28}$ \\ (Affiliations are available in the online edition)
}

Received 1 April 2010 / Accepted 18 May 2010

\begin{abstract}
The Herschel Lensing Survey (HLS) takes advantage of gravitational lensing by massive galaxy clusters to sample a population of high-redshift galaxies which are too faint to be detected above the confusion limit of current far-infrared/submillimeter telescopes. Measurements from 100-500 $\mu \mathrm{m}$ bracket the peaks of the far-infrared spectral energy distributions of these galaxies, characterizing their infrared luminosities and star formation rates. We introduce initial results from our science demonstration phase observations, directed toward the Bullet cluster (1E0657-56). By combining our observations with LABOCA $870 \mu \mathrm{m}$ and AzTEC $1.1 \mathrm{~mm}$ data we fully constrain the spectral energy distributions of 19 MIPS $24 \mu \mathrm{m}$-selected galaxies which are located behind the cluster. We find that their colors are best fit using templates based on local galaxies with systematically lower infrared luminosities. This suggests that our sources are not like local ultra-luminous infrared galaxies in which vigorous star formation is contained in a compact highly dust-obscured region. Instead, they appear to be scaled up versions of lower luminosity local galaxies with star formation occurring on larger physical scales.
\end{abstract}

Key words. infrared: galaxies - submillimeter: galaxies - galaxies: evolution - galaxies: high-redshift - galaxies: clusters: general gravitational lensing: strong

\section{Introduction}

Luminous (and ultra-luminous) infrared galaxies ((U)LIRGS) are a population of massive star-forming galaxies which contain significant amounts of dust, absorbing the ultraviolet emission from newly formed stars within them and re-radiating this energy at far-infrared (FIR) wavelengths. The FIR emission of these sources is therefore strongly correlated with their level of star formation activity (e.g. Kennicutt 1998). Spitzer MIPS measurements from 24-160 $\mu \mathrm{m}$ bracket the peak of the dust emission from nearby (U)LIRGS. Therefore, MIPS observations have been very successful in characterizing the spectral energy distributions (SEDs) and star formation rates (SFRs) of the local population (e.g. Rieke et al. 2009). Similar measurements at FIR/submillimeter (submm) wavelengths are required to constrain the SEDs of their higher redshift counterparts. Together the PACS (Poglitsch et al. 2010) and SPIRE (Griffin et al. 2010)

* Herschel is an ESA space observatory with science instruments provided by European-led Principal Investigator consortia and with important participation from NASA. Data presented in this paper were analyzed using "The Herschel interactive processing environment (HIPE)", a joint development by the Herschel Science Ground Segment Consortium, consisting of ESA, the NASA Herschel Science Center, and the HIFI, PACS, and SPIRE consortia.

$\star \star$ Table 1 and Figs. 3, 4 are only available in electronic form at http://www . aanda. org instruments on-board Herschel (Pilbratt et al. 2010) provide sensitive measurements of this population at five wavelengths from 100-500 $\mu \mathrm{m}$, sampling the rest-frame peak of the FIR emission out to a redshift of $z=4$.

The depth of submm maps is ultimately limited by confusion noise resulting from a high density of sources relative to the angular resolution of the telescope. Gravitational lensing by massive galaxy clusters provides the only means to sample an abundant population of intrinsically faint or high-redshift infrared (IR) galaxies which lie below this limit. The Herschel Lensing Survey (HLS) (Egami et al. 2010) will target 40 galaxy clusters with PACS and SPIRE to compile the first significant submm catalog of these galaxies. In this letter we present initial results from our science demonstration phase observations: $8^{\prime} \times 8^{\prime}$ PACS maps and $17^{\prime} \times 17^{\prime}$ SPIRE maps centered on the Bullet cluster (1E0657-56). Details of these observations are presented in Egami et al. (2010). This is a unique target because of its strong lensing potential and the richness of existing ancillary data. We present the FIR properties of 19 MIPS $24 \mu \mathrm{m}$-selected galaxies which are located behind the Bullet cluster. We compare the shapes of their SEDs with those of local (U)LIRGS, and compare their measured IR luminosities with predictions which extrapolate this quantity from the observed MIPS $24 \mu \mathrm{m}$ flux. Subsequent uses of MIPS in the text refer to the MIPS $24 \mu \mathrm{m}$ band. 


\section{Data and source catalog}

The PACS and SPIRE observations of the Bullet cluster were reduced using the Herschel interactive processing environment (HIPE). Small deviations from the standard pipeline are discussed in Egami et al. (2010).

\subsection{Source selection}

PACS and SPIRE data provide unprecedented sensitivities at FIR/submm wavelengths. Nonetheless, the precise identification of IR galaxies based solely on Herschel maps is complicated due to relatively large beam sizes leading to confusion noise in the SPIRE bands. We have therefore constructed an initial catalog with the positions of IR sources in the region based on secure $(>10-\sigma)$ detections in the higher resolution Spitzer MIPS $24 \mu \mathrm{m}$ map of the field. A $10-\sigma 24 \mu \mathrm{m}$ threshold corresponds to the 1- $\sigma$ error in the PACS $100 \mu \mathrm{m}$ map, our deepest Herschel map of the field. We select the positions for our photometry from this MIPS catalog in an effort to minimize the effect of Eddington bias which artificially boosts the flux of sources selected from confused submillimeter maps because of the steep underlying number counts. A proper statistical treatment of this effect will be presented in the more comprehensive analysis of this field in preparation.

In order to identify a subset of these initial sources which lie behind the Bullet cluster, we have compiled all of the spectroscopic redshift information for galaxies in the field obtained thus far. A description of these redshift catalogs and our method of association with the MIPS positions is given in Rawle et al. (2010). A histogram of the redshifts reveals a large group of Bullet cluster members at $z \sim 0.3$, as well as a smaller cluster of galaxies located just beyond at $z \sim 0.35$ (Rawle et al. 2010). We have therefore chosen a lower limit of $z \geq 0.40$ to select background field galaxies. With these criteria we assemble a subcatalog of 50 MIPS-selected galaxies which are spectroscopically confirmed to be located behind the Bullet cluster. In many cases one SPIRE beam contains more than one MIPS galaxy. In these instances we are sometimes able to use the PACS resolution to identify the dominant source of the SPIRE emission. If this is not possible we exclude the source from our present analysis. Finally, we constrain our work to sources with $>3-\sigma$ detections in at least two Herschel bands. We are thereby left with a sample of 15 significant background galaxies with spectroscopic redshifts.

We also present the analysis of 4 additional galaxies selected because of photometric redshift (photo-z) estimates suggesting they are at $z_{\text {phot }}>1.5$, and which are detected in LABOCA $870 \mu \mathrm{m}$ (Johansson et al. 2010) and AzTEC $1.1 \mathrm{~mm}$ maps of the field (Wilson et al. 2008). We have calculated photo-z's for these sources using two methods: one based on IRAC colors (Pérez-González et al. 2005) and the other on the FIR - mm colors (Hughes et al. 2002; Aretxaga et al. 2003). When the two estimates disagree we have chosen the value which yields the lowest $\chi^{2}$ fit to our SED templates. Including these galaxies in our analysis allows us to take advantage of the substantial correlation between SPIRE maps and longer wavelength submm/mm maps of the field. These results demonstrate the strength of combining such data sets to identify a potentially higher redshift galaxy population. The positions of our selections, along with corresponding MIPS and Herschel photometry can be found in Table 1 (included in the online supplementary material).

\subsection{Photometry}

Herschel photometry for these galaxies is measured by simultaneously fitting the PSF at the positions of all of the 10- $\sigma$ MIPS sources identified in our original catalog using routines included in the IRAF package DAOPHOT. To ensure the maps are aligned to the same astrometry small offsets are applied to the PACS data based on the results of stacking the $100 \mu \mathrm{m}$ map on the MIPS positions and to the SPIRE data based on the results of stacking the $250 \mu \mathrm{m}$ map on the MIPS positions. The $500 \mu \mathrm{m}$ map is also corrected for contamination from the Sunyaev-Zel'dovich effect based on fits to the data presented in Zemcov et al. (2010) before performing photometry.

In cases where multiple MIPS galaxies fall within half the beam size at the corresponding waveband, we approximate the position of the group by the average of the counterparts weighted by their $24 \mu \mathrm{m}$ signal-to-noise. We then take an iterative approach; fitting at the positions of the brightest sources first, removing them from the map, fitting at the positions of the next tier, and so on until we have reached the 3- $\sigma$ noise level of the observation. This method is similar to that outlined in Pérez-González et al. (2010), although we force the algorithm to fit the PSF at the MIPS positions rather than allowing for any re-centering.

Our analysis includes LABOCA $870 \mu \mathrm{m}$ and AzTEC $1.1 \mathrm{~mm}$ photometry for our sources when it is of $>3-\sigma$ significance. Four of our objects have LABOCA counterparts within $8^{\prime \prime}$ listed in the photometric catalog from Johansson et al. (2010). In these cases we use the deboosted flux given in the catalog for our analysis. In order to obtain photometry for the remaining sources, we measure the flux in an aperture of $40^{\prime \prime}$ at the MIPS position in order to get the total flux from the beam. This method is not the same as that used in Johansson et al. (2010), although it gives consistent results for the 17 sources presented therein. In the case of the four sources chosen, in part, due to bright longwavelength emission, we identify clear associations between the MIPS source and a significant, individual AzTEC source. In these cases we use the deboosted flux from the AzTEC cata$\log$. For the other sources the AzTEC fluxes are measured at the MIPS positions from a PSF-convolved map. In the instances where there is a counterpart (within $8^{\prime \prime}$ ) in the AzTEC catalog, the difference between the flux at the MIPS position and the deboosted point-source flux is $<10 \%$.

\section{Results}

Figure 1 shows the most highly magnified galaxy in our sample, first reported as a strongly lensed IRAC source (Bradač et al. 2006; Gonzalez et al. 2009) and independently found to be the brightest submm/mm source in the field (Wilson et al. 2008; Rex et al. 2009; Johansson et al. 2010). Multiple images of the galaxy are indicated with white circles. The right panel shows our SED fit to the sum of the fluxes from the brightest of the two images. SPIRE data indicate a $250 \mu \mathrm{m}$ flux density which is $30 \%$ lower than that measured in the BLAST data (Rex et al. 2009), although the measurements are consistent within the uncertainties. The Spitzer InfraRed Spectrograph (IRS) spectrum of this source confirms that it is at redshift $z=2.79$ (Gonzalez et al. 2010), consistent with published photo- $z$ estimates similar to the ones used for the four sources in our sample which lack spectroscopic redshift information. At that redshift, our model indicates a magnification of $\sim 50$ due to gravitational lensing by the foreground cluster. This value is lower than other estimates presented in the literature (e.g. Gonzalez et al. 2010 

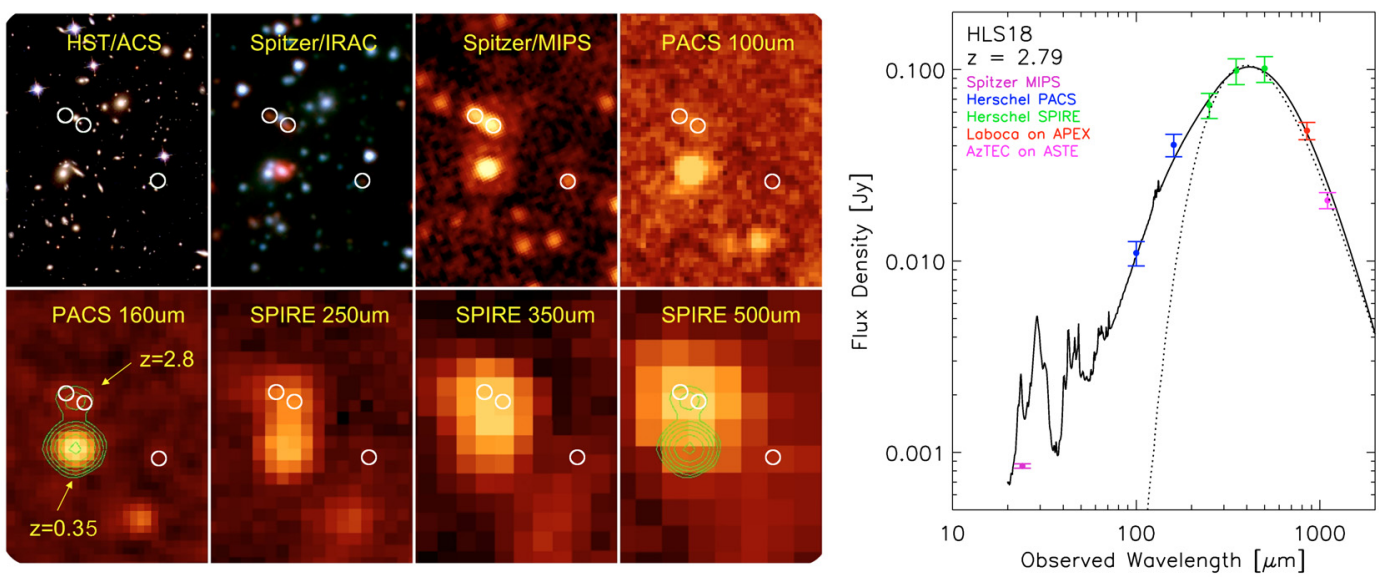

Fig. 1. The most highly magnified galaxy in our sample, HLS18, is the same LIRG first detected by Wilson et al. (2008) with AzTEC at $1.1 \mathrm{~mm}$. Left panel: $1.5^{\prime} \times 2^{\prime}$ thumbnails of the source at a variety of wavelengths highlight the importance of multi-wavelength data to guide the interpretation of the lower resolution maps. IRAC data reveal three lensed images of the galaxy indicated with white circles, although only emission from the two brightest images is detected in the Herschel maps. Right panel: Rieke et al. (2009) SED template fit to the source. The fluxes include the contribution from both of the brightest images of the galaxy, indicated by the two left-most circles in the thumbnails. The data points have not been de-magnified. The $24 \mu \mathrm{m}$ flux is plotted for reference, although it was not used in the fit. The best-fit modified blackbody is shown with a dotted line and is consistent with previous estimates (Wilson et al. 2008; Gonzalez et al. 2009; Rex et al. 2009).

suggest a magnification of $\sim 100)$. We note that this value should be treated as a lower limit since there is likely to be additional local lensing from nearby objects. We derive an observed IR luminosity of $2.8 \times 10^{13} L_{\odot}$ based on the template SED fitting (see below for a detailed discussion of this SED fitting procedure). Adopting our estimated lower limit for the magnification of the source indicates an intrinsic IR luminosity of $\$ 5 \times 10^{11} L_{\odot}$ which is consistent with calculations previously reported in the literature (Wilson et al. 2008; Gonzalez et al. 2009; Rex et al. 2009; Gonzalez et al. 2010), apart from the assumed magnification factor. The detection of such an intrisically faint galaxy is not possible without gravitational lensing, clearly demonstrating the power and promise of our strategy.

\subsection{SED properties}

The galaxies in our sample span a redshift range of $0.40<z<$ 3.24. Knowing their redshifts, we can derive their apparent IR luminosities. We use the lensing models described in Paraficz et al. (in prep.) to correct for the magnification of each source due to gravitational lensing by the foreground cluster and calculate the galaxies' intrinsic properties. In our discussion of IR properties, we adopt nomenclature similar to that in Rieke et al. (2009), defining the total IR luminosity $\left(L_{\mathrm{TIR}}\right)$ as the luminosity in the rest-frame wavelength range $\lambda=5-1000 \mu \mathrm{m}$. The redshifts, magnifications, and de-magnified IR luminosities of each source are listed in Table 2 . We assume a $\Lambda$ CDM cosmology with $\Omega_{\mathrm{m}}=0.3, \Omega_{\Lambda}=0.7$, and $H_{0}=70 \mathrm{~km} \mathrm{~s}^{-1} \mathrm{Mpc}^{-1}$ for our calculations.

We use a $\chi^{2}$ minimization routine to fit the SED templates presented in Rieke et al. (2009) (R09), as well as those presented in Chary \& Elbaz (2001) (CE01). These templates are based on data from local galaxies and each is designated by a luminosity class corresponding to the total infrared luminosities of the local (U)LIRGS for which it provides the best fit. The left panel of Fig. 2 shows an example of the template fits to a galaxy in our sample. The solid lines show the best-fit R09 template (in blue) and CE01 template (in red) to the FIR-mm data, excluding the $24 \mu \mathrm{m}$ point. The dotted lines show the respective fits to only the observed $24 \mu \mathrm{m}$ point. The SED fits to the remaining galaxies in our sample are shown in Figs. 3 and 4 (included in the online supplementary material).

In general we find that the templates provide good fits to the FIR-mm data in our galaxy sample and that the IR luminosities derived using the best-fit templates from both R09 and CE01 agree within a $1-\sigma$ spread of $15 \%$. We also find that the galaxies in our sample are best fit by templates with systematically lower luminosity classes. The top right panels (b and c) of Fig. 2 illustrate this trend. The luminosity class of the bestfit template to each galaxy is plotted as a function of the actual luminosity for the R09 templates in (b) and the CE01 templates in (c). Although we find a large scatter in the luminosity classes of best-fit SEDs, both template families show the same general trend. This is because the SEDs of our galaxies peak at longer wavelengths than local galaxies of comparable luminosities. Therefore their spectral shapes more closely resemble those of lower luminosity (U)LIRGS in the local universe. The result supports evidence that high-redshift submm galaxies are cooler than local galaxies with similar IR luminosities, suggesting the star formation within them is occurring on more extended physical scales (e.g. Chapman et al. 2004).

The discrepancy between the FIR SED shapes of our sources and those of local counterparts with similar luminosities implies that a blind application of the local template SED models might not accurately predict the $L_{\mathrm{TIR}}$ and hence the SFRs of higher redshift submm galaxies. However, other studies based on Herschel data show that the $L_{\mathrm{TIR}}$ predicted by observed $24 \mu \mathrm{m}$ emission is in good agreement with that measured from the FIR data for galaxies at $z<1.5$ (Elbaz et al. 2010). The bottom right panels of Fig. 2 confirm this trend. The IR luminosity derived from $24 \mu \mathrm{m}$ is plotted as a function of the actual IR luminosity for the R09 templates in (d) and the CE01 templates in (e). Although the R09 fits show a slightly higher deviation, both template families predict reasonable values for the luminosities of the galaxies in our sample, except in the cases of the ULIRGS/Hyper-LIRGS at $z>1.5$. The left panel of Fig. 2 shows a closer inspection of the SED fits, revealing that although the predicted IR luminosities are consistant with the measured values, the SED templates selected based solely on observed $24 \mu \mathrm{m}$ emission typically peak at shorter wavelengths, and do not provide good fits to the FIR-mm data. 

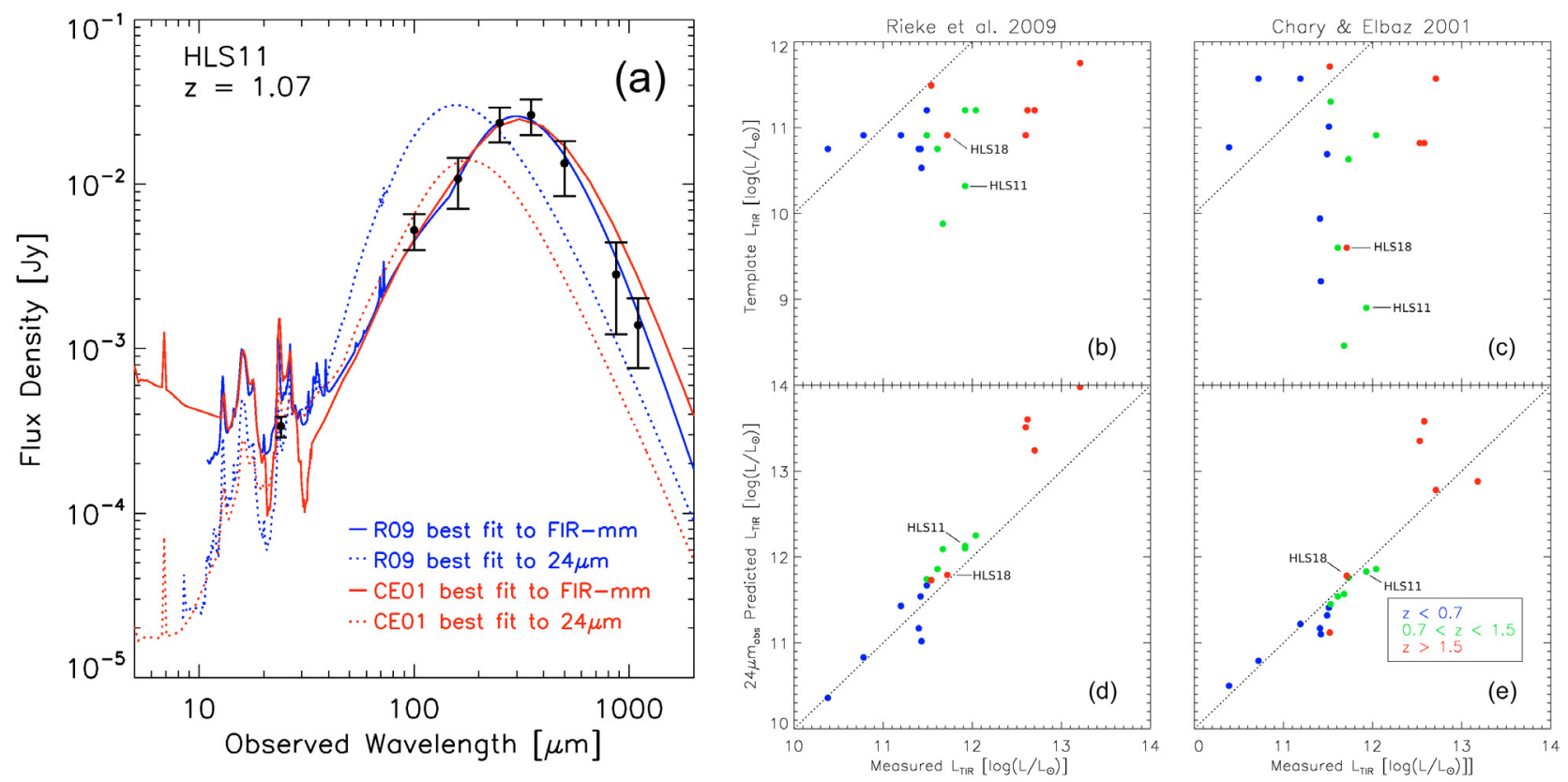

Fig. 2. Left panel a): SED template fits to HLS11, a redshift $z \sim 1$ LIRG. The data points have been de-magnified by a factor of 1.4 based on our lensing models of the foreground cluster. Although both the R09 and the CE01 templates predict the IR luminosity based on observed $24 \mu \mathrm{m}$ emission to within a factor of 2 , the $24 \mu \mathrm{m}$-predicted SEDs do not provide reasonable fits to the FIR-mm data. Right top panels b) and c): the IR luminosity of the best-fit template as it corresponds to the local population is plotted as a function of the actual IR luminosity of each galaxy. The SEDs of our sample are better fit by templates corresponding to systematically lower luminosity local galaxies. Right bottom panels $\mathbf{d}$ ) and e): the $24 \mu \mathrm{m}$-predicted IR luminosity is plotted as a function of the actual IR luminosity of each galaxy. Both template families predict reasonable values for the luminosities of the galaxies in our sample, except in the cases of the ULIRGS/Hyper-LIRGS at $z>1.5$. Note that HLS11 \& HLS18 (Fig. 1) fall on the trend line even though their SED shapes show significant deviation in b) and $\mathbf{c}$ ).

Table 2. Source properties.

\begin{tabular}{|c|c|c|c|}
\hline ID & $z$ & Mag & $\mathrm{R} 09 L_{\mathrm{TIR}}\left[L_{\odot}\right]$ \\
\hline HLS01 & 0.61 & 1.1 & $2.6 e+11$ \\
\hline HLS02 & 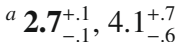 & 1.00 & $4.0 \mathrm{e}+12$ \\
\hline HLS03 & 0.72 & 1.0 & $3.1 \mathrm{e}+11$ \\
\hline HLS04 & 0.82 & 1.0 & $4.1 e+11$ \\
\hline HLS05 & ${ }^{a} \mathbf{1 . 6}^{+.1}, \mathbf{1 . 6}^{+.4}$ & 1.16 & $1.6 e+13$ \\
\hline HLS06 & a $2.8_{-1}^{+.1}, \mathbf{1 . 8}_{-4}^{+.5}$ & 1.11 & $5.0 \mathrm{e}+12$ \\
\hline HLS07 & 0.66 & 1.1 & $1.6 e+11$ \\
\hline HLS08 & 0.43 & 1.2 & $2.4 e+10$ \\
\hline HLS09 & 0.82 & 1.1 & $8.3 e+11$ \\
\hline HLS10 & 0.60 & 1.1 & $3.1 e+11$ \\
\hline HLS11 & 1.07 & 1.4 & $8.3 e+11$ \\
\hline HLS12 & 3.24 & 11.3 & $3.5 e+11$ \\
\hline HLS13 & ${ }^{a} \mathbf{2 . 9} \mathbf{9}_{-1}^{+.1}, 3.1_{-3}^{+.7}$ & 1.23 & $4.2 \mathrm{e}+12$ \\
\hline HLS14 & 0.57 & 1.1 & $6.0 \mathrm{e}+10$ \\
\hline HLS15 & 1.17 & 1.1 & $4.7 e+11$ \\
\hline HLS16 & 0.45 & 1.4 & $2.5 e+11$ \\
\hline HLS17 & 0.40 & 1.1 & $2.7 e+11$ \\
\hline HLS18 & 2.79 & $>54^{b}$ & $5.2 e+11$ \\
\hline HLS19 & 0.71 & 1.1 & $1.1 \mathrm{e}+12$ \\
\hline
\end{tabular}

Notes. ${ }^{(a)}$ IRAC photo- $z$, FIR-mm photo- $z$. Adopted value in bold; (b) lower limit due to unquantified local lensing by nearby objects.

\section{Conclusion}

We have presented the first Herschel analysis of galaxies located behind the Bullet cluster. We find that their colors are best fit using templates based on local galaxies with systematically lower IR luminosities. This suggests that our sources are not like local ULIRGS in which vigorous star formation is contained in a compact highly dust-obscured region.
Instead, they appear to be scaled up versions of lower luminosity local galaxies with star formation occurring on larger physical scales. A more comprehensive analysis of the field in preparation will combine our full catalog of sources with LABOCA and AzTEC data to compile a larger sample of these galaxies. By studying their relationship to local star-forming galaxies we can better understand the processes that govern their evolution.

Acknowledgements. We thank Ben Weiner and David Elbaz for their valuable comments and help with our computations. This work is based in part on observations made with Herschel, a European Space Agency Cornerstone Mission with significant participation by NASA. Support for this work was provided by NASA through an award issued by JPL/Caltech.

\section{References}

Aretxaga, I., Hughes, D. H., Chapin, E. L., et al. 2003, MNRAS, 342, 759 Bradač, M., Clowe, D., Gonzalez, A. H., et al. 2006, ApJ, 652, 937

Chapman, S. C., Smail, I., Windhorst, R., Muxlow, T., \& Ivison, R. J. 2004, ApJ, 611,732

Chary, R., \& Elbaz, D. 2001, ApJ, 556, 562

Egami, E., et al. 2010, A\&A, 518, L12

Elbaz, D., et al. 2010, A\&A, 518, L29

Gonzalez, A. H., Clowe, D., Bradač, M., et al. 2009, ApJ, 691, 525

Gonzalez, A. H., Papovich, D., Bradač, M., \& Jones, C. 2010, ApJ, submitted Griffin, M. J., et al. 2010, A\&A, 518, L3

Hughes, D. H., Aretxaga, I., Chapin, E. L., et al. 2002, MNRAS, 335, 871

Johansson, D., Horellou, C., Sommer, M. W., et al. 2010, A\&A, 514, A77 Kennicutt, Jr., R. C. 1998, ApJ, 498, 541

Pérez-González, P. G., Rieke, G. H., Egami, E., et al. 2005, ApJ, 630, 82

Pérez-González, P. G., et al. 2010, A\&A, 518, L15

Pilbratt, G. L., et al. 2010, A\&A, 518, L1

Poglitsch, A., et al. 2010, A\&A, 518, L2

Rawle, T. D., et al. 2010, A\&A, 518, L14

Rex, M., Ade, P. A. R., Aretxaga, I., et al. 2009, ApJ, 703, 348

Rieke, G. H., Alonso-Herrero, A., Weiner, B. J., et al. 2009, ApJ, 692, 556

Wilson, G. W., Hughes, D. H., Aretxaga, I., et al. 2008, MNRAS, 390, 1061

Zemcov, M., et al. 2010, A\&A, 518, L16

\section{Pages 5 to 8 are available in the electronic edition of the journal at http://www . aanda. org}


1 Steward Observatory, University of Arizona, 933 N. Cherry Ave, Tucson, AZ 85721, USA

e-mail: mrex@as.arizona.edu

2 Departamento de Astrofísica, Facultad de CC. Físicas, Universidad Complutense de Madrid, 28040 Madrid, Spain

${ }^{3}$ California Institute of Technology, Pasadena, CA 91125, USA

4 Jet Propulsion Laboratory, Pasadena, CA 91109, USA

5 Instituto Nacional de Astrofísica Óptica y Electrónica (INAOE), Luis Enrique Erro No.1, Tonantzintla, Puebla, CP 72840, Mexico

${ }^{6}$ Department of Astronomy, University of Florida, Gainesville, FL 32611-2055, USA

7 NASA Herschel Science Center, California Institute of Technology, MS 100-22, Pasadena, CA 91125, USA

8 Onsala Space Observatory, Chalmers University of Technology, 43992 Onsala, Sweden

${ }^{9}$ Laboratoire d'Astrophysique de Marseille, CNRS - Université AixMarseille, 38 Rue Frédéric Joliot-Curie, 13388 Marseille Cedex 13, France

10 Institute for Computational Cosmology, Department of Physics, Durham University, South Road, Durham DH1 3LE, UK

11 Herschel Science Centre, ESAC, ESA, PO Box 78, Villanueva de la Cañada, 28691 Madrid, Spain

12 Laboratoire d'Astrophysique de Toulouse-Tarbes, Université de Toulouse, CNRS, 14 Av. Edouard Belin, 31400 Toulouse, France

13 Observatoire de Paris, LERMA, 61 Av. de l'Observatoire, 75014 Paris, France

14 Geneva Observatory, University of Geneva, 51, Ch. des Maillettes, 1290 Versoix, Switzerland
15 UK Astronomy Technology Centre, Science and Technology Facilities Council, Royal Observatory, Blackford Hill, Edinburgh EH9 3HJ, UK

16 Institute for Astronomy, University of Edinburgh, Blackford Hill, Edinburgh EH9 3HJ, UK

17 Max-Planck-Institut für extraterrestrische Physik, Postfach 1312, 85741 Garching, Germany

18 Institut d'Astrophysique de Paris, CNRS and Université Pierre et Marie Curie, 98bis Boulevard Arago, 75014 Paris, France

19 Department of Astronomy, University of Padova, Vicolo dell'Osservatorio 3, 35122 Padova, Italy

20 School of Physics and Astronomy, University of Birmingham, Edgbaston, Birmingham, B15 2TT, UK

21 Sterrewacht Leiden, Leiden University, PO Box 9513, 2300 RA Leiden, The Netherlands

22 Center for Astrophysics and Space Astronomy, University of Colorado, Boulder, CO 80309, USA

23 Nobeyama Radio Observatory, National Astronomical Observatory of Japan, Minamimaki, Minamisaku, Nagano 384-1305, Japan

24 Institute of Astronomy, University of Tokyo, 2-21-1 Osawa, Mitaka, Tokyo 181-0015, Japan

25 Research Center for the Early Universe, School of Science, University of Tokyo, 7-3-1 Hongo, Bunkyo, Tokyo 113-0033, Japan

26 Department of Physics, Illinois Wesleyan University, Bloomington, IL 61702-2900, USA

27 Department of Physics \& Astronomy, University of Pennsylvania, 209 South 33rd Street, Philadelphia, PA 19104, USA

28 Department of Astronomy, University of Massachusetts, 710 North Pleasant Street, Amherst, MA 01003, USA 
Table 1. Flux densities measured from the MIPS and Herschel maps.

\begin{tabular}{lllllllll}
\hline \hline ID & RA & $\begin{array}{l}\text { Dec } \\
{[\mathrm{deg}]}\end{array}$ & $\begin{array}{l}\text { Flux }_{24} \\
{[\mathrm{mJy}]}\end{array}$ & $\begin{array}{l}\text { Flux }_{100} \\
{[\mathrm{mJy}]}\end{array}$ & $\begin{array}{l}\text { Flux }_{160} \\
{[\mathrm{mJy}]}\end{array}$ & $\begin{array}{l}\text { Flux }_{250} \\
{[\mathrm{mJy}]}\end{array}$ & $\begin{array}{l}\text { Flux }_{350} \\
{[\mathrm{mJy}]}\end{array}$ & $\begin{array}{l}\text { Flux }_{500} \\
{\left[\mathrm{mJy}^{2}\right.}\end{array}$ \\
\hline HLS01 & 104.72828 & -55.88917 & $0.40 \pm 0.01$ & $\ldots$ & $\ldots$ & $21.1 \pm 2.5$ & $10.0 \pm 3.5$ & $<7.8^{b}$ \\
HLS02 & 104.46419 & -56.02209 & $0.15 \pm 0.01$ & $\ldots$ & $\ldots$ & $10.1 \pm 2.4$ & $16.2 \pm 3.5$ & $14.4 \pm 4.0$ \\
HLS03 & 104.48783 & -56.03070 & $0.39 \pm 0.02$ & $\ldots$ & $\ldots$ & $9.0 \pm 2.4$ & $9.5 \pm 3.4$ & $<7.8^{b}$ \\
HLS04 & 104.45727 & -55.91533 & $0.39 \pm 0.01$ & $\ldots$ & $\ldots$ & $18.6 \pm 2.8$ & $13.2 \pm 3.6$ & $<7.8^{b}$ \\
HLS05 & 104.60260 & -55.92013 & $0.71 \pm 0.02$ & $75.4 \pm 1.3$ & $164.4 \pm 5.1$ & $168.9 \pm 6.2$ & $120.0 \pm 4.1$ & $58.4 \pm 4.4$ \\
HLS06 & 104.64300 & -55.90990 & $0.40 \pm 0.01$ & $9.9 \pm 1.2$ & $28.4 \pm 2.4$ & $44.6 \pm 2.8$ & $39.0 \pm 3.5$ & $25.2 \pm 4.0$ \\
HLS07 & 104.60545 & -55.89051 & $0.29 \pm 0.01$ & $7.0 \pm 1.3$ & $7.8 \pm 2.1$ & $8.2 \pm 2.8$ & $<8.4^{b}$ & $<7.8^{b}$ \\
HLS08 & 104.63628 & -55.98351 & $0.135 \pm 0.003$ & $2.7 \pm 1.1$ & $4.6 \pm 2.1$ & $<6.0^{b}$ & $<8.4^{b}$ & $<7.8^{b}$ \\
HLS09 & 104.55591 & -55.87279 & $0.65 \pm 0.02$ & $\ldots$ & $\ldots$ & $23.2 \pm 2.6$ & $12.9 \pm 3.5$ & $<7.8^{b}$ \\
HLS10 & 104.54350 & -55.98005 & $0.53 \pm 0.02$ & $16.7 \pm 1.4$ & $22.6 \pm 2.1$ & $13.9 \pm 2.4$ & $<8.4^{b}$ & $<7.8^{b}$ \\
HLS11 & 104.56008 & -55.95848 & $0.47 \pm 0.01$ & $7.4 \pm 1.1$ & $15.1 \pm 2.1$ & $33.1 \pm 2.7$ & $36.9 \pm 3.5$ & $18.8 \pm 4.1$ \\
HLS12 & 104.62997 & -55.94386 & $0.046 \pm 0.003$ & $<2.4^{b}$ & $4.3 \pm 2.1$ & $7.8 \pm 2.5$ & $11.4 \pm 3.4$ & $<7.8^{b}$ \\
HLS13 & 104.60567 & -55.94490 & $0.28 \pm 0.01$ & $2.9 \pm 1.1$ & $5.5 \pm 2.0$ & $10.1 \pm 2.5$ & $19.6 \pm 3.5$ & $<7.8^{b}$ \\
HLS14 & 104.64689 & -55.88658 & $0.136 \pm 0.004$ & $3.0 \pm 1.2$ & $4.4 \pm 2.1$ & $<6.0^{b}$ & $<8.4^{b}$ & $<7.8^{b}$ \\
HLS15 & 104.73730 & -55.88516 & $0.16 \pm 0.02$ & $\ldots$ & $\ldots$ & $11.1 \pm 2.7$ & $15.3 \pm 3.8$ & $11.7 \pm 3.7$ \\
HLS16 & 104.58575 & -55.93920 & $0.83 \pm 0.02$ & $25.3 \pm 1.3$ & $51.3 \pm 2.5$ & $47.7 \pm 2.6$ & $24.0 \pm 3.6$ & $10.2 \pm 3.7$ \\
HLS17 & 104.64463 & -56.00850 & $0.71 \pm 0.02$ & $24.7 \pm 1.2$ & $56.5 \pm 3.4$ & $49.2 \pm 3.4$ & $35.5 \pm 3.7$ & $17.9 \pm 3.7$ \\
HLS18 ${ }^{a}$ & 104.65471 & -55.95193 & $0.49 \pm 0.01$ & $7.0 \pm 1.2$ & $24.5 \pm 2.1$ & $65.3 \pm 2.8$ & $98.6 \pm 3.9$ & $101.4 \pm 4.0$ \\
HLS18 ${ }^{a}$ & 104.65861 & -55.95057 & $0.36 \pm 0.01$ & $3.8 \pm 1.1$ & $14.0 \pm 2.5$ & $<6.0^{b}$ & $<8.4^{b}$ & $<7.8^{b}$ \\
HLS19 & 104.59877 & -55.87833 & $1.10 \pm 0.03$ & $\ldots$ & $\ldots$ & $40.9 \pm 2.9$ & $21.6 \pm 4.0$ & $9.4 \pm 3.7$ \\
\hline
\end{tabular}

Notes. The $500 \mu \mathrm{m}$ map was corrected for SZ contamination before extracting the photometry. These measurements have not been corrected for the amplification due to gravitational lensing. ${ }^{(a)}$ Multiple images of the highly lensed galaxy (Gonzalez et al. 2009); ${ }^{(b)}$ upper limits correspond to statistical 3- $\sigma$ noise levels of each map. 
M. Rex et al.: The far-infrared/submillimeter properties of galaxies located behind the Bullet cluster
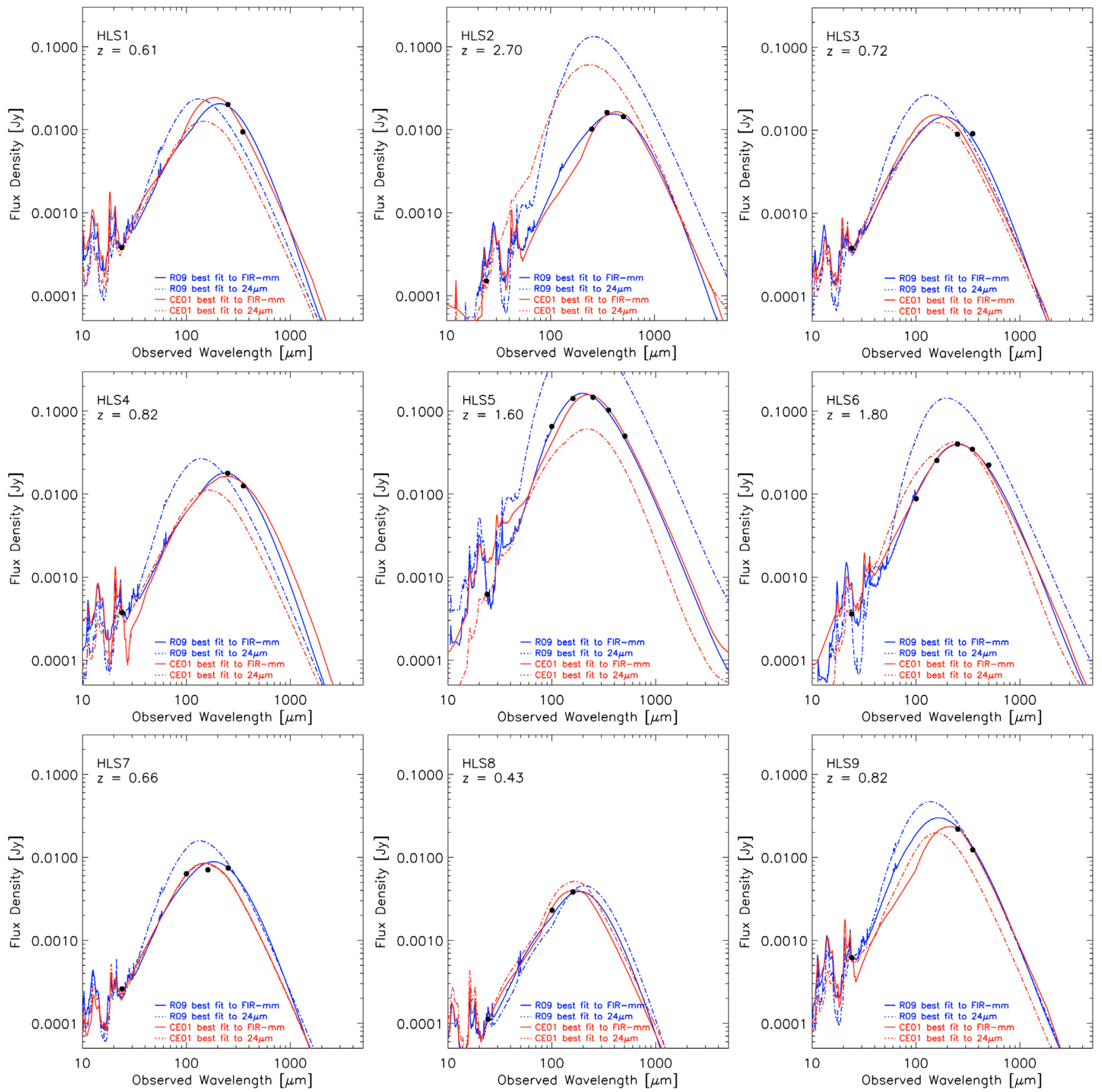

Fig. 3. SED template fits to HLS1-HLS9. The data points have been de-magnified according to our lensing models of the foreground cluster. These magnification factors can be found in Table 2. The solid lines show the best-fit R09 template (in blue) and CE01 template (in red) to the FIR-mm data, excluding the $24 \mu \mathrm{m}$ point. LABOCA $870 \mu \mathrm{m}$ and AzTEC $1.1 \mathrm{~mm}$ data are used to constrain these fits when the detection is of $>3-\sigma$ significance. However, only the MIPS and Herschel data are shown in the figure. The dotted lines show the respective fits based solely on the observed $24 \mu \mathrm{m}$ point. Although both the R09 and the CE01 templates generally predict reasonable values for the IR luminosity based on observed $24 \mu \mathrm{m}$ emission, the $24 \mu \mathrm{m}$-predicted SEDs typically do not provide reasonable fits to the FIR-mm data. 

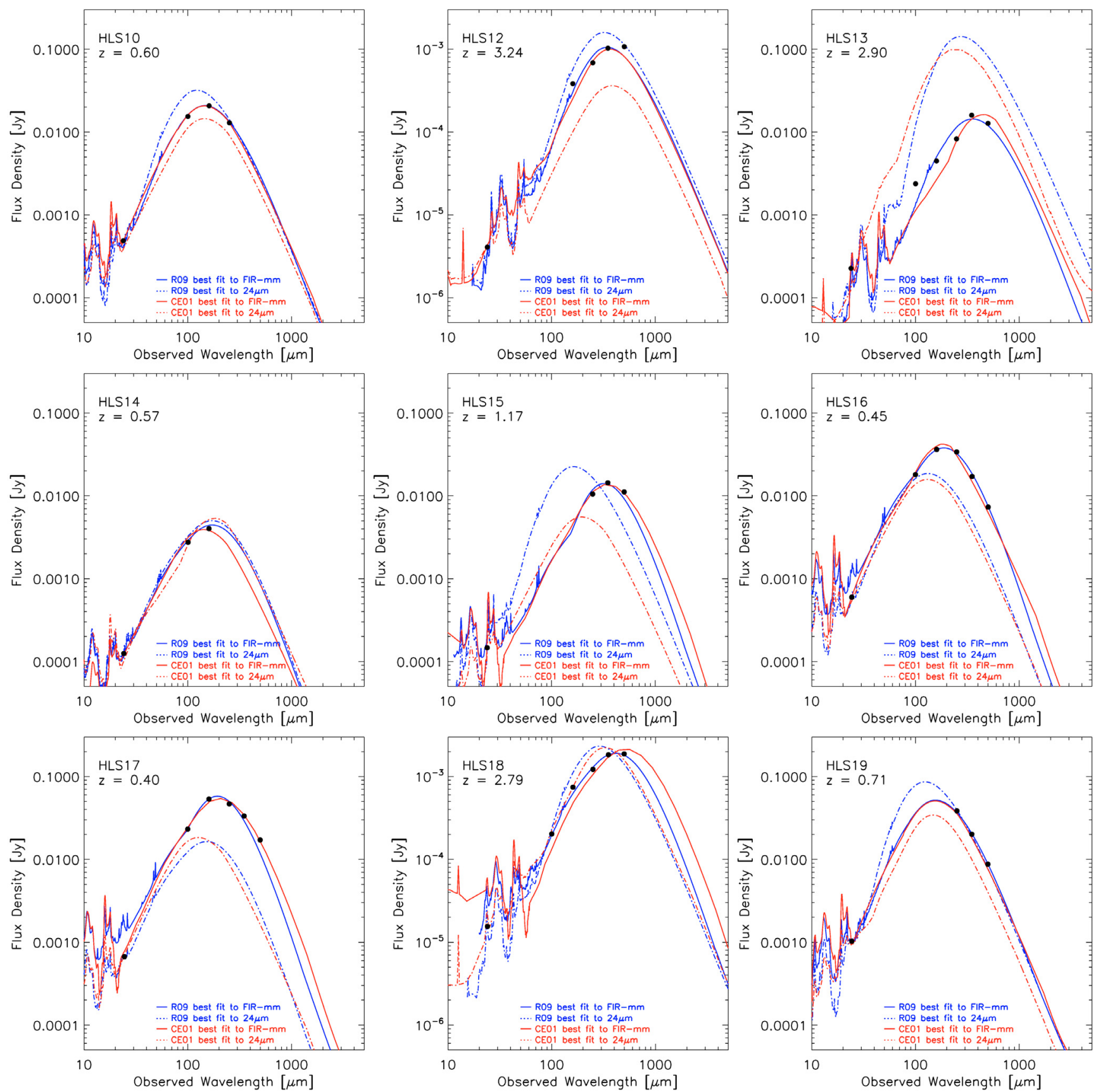

Fig. 4. SED template fits to HLS10-HLS19. The data points have been de-magnified according to our lensing models of the foreground cluster. These magnification factors can be found in Table 2. The solid lines show the best-fit R09 template (in blue) and CE01 template (in red) to the FIR-mm data, excluding the $24 \mu \mathrm{m}$ point. LABOCA $870 \mu \mathrm{m}$ and AzTEC $1.1 \mathrm{~mm}$ data are used to constrain these fits when the detection is of $>3-\sigma$ significance. However, only the MIPS and Herschel data are shown in the figure. The dotted lines show the respective fits based solely on the observed $24 \mu \mathrm{m}$ point. Although both the R09 and the CE01 templates generally predict reasonable values for the IR luminosity based on observed $24 \mu \mathrm{m}$ emission, the $24 \mu \mathrm{m}$-predicted SEDs typically do not provide reasonable fits to the FIR-mm data. 